\title{
Integralidade e Atenção Primária à Saúde: avaliação sob a ótica dos usuários
}

\author{
The comprehensive nature of primary health care: \\ evaluation from the user standpoint
}

\author{
Carla Silvana de Oliveira e Silva ${ }^{1}$ \\ Adélia Dayane Guimarães Fonseca ${ }^{2}$ \\ Luís Paulo Souza e Souza ${ }^{3}$ \\ Leila das Graças Siqueira ${ }^{3}$ \\ Angélica Gonçalves Silva Belasco ${ }^{1}$ \\ Dulce Aparecida Barbosa ${ }^{1}$
}

${ }^{1}$ Programa de PósGraduação em Ciências, Escola Paulista de Enfermagem, Universidade Federal de São Paulo. R. Napoleão de Barros 754, Vila Clementino. 04024002 São Paulo SP Brasil. carlasosilva@ig.com.br ${ }^{2}$ Departamento de Enfermagem, Faculdade de Saúde e Desenvolvimento Humano Santo Agostinho. ${ }^{3}$ Departamento de Enfermagem, Universidade Estadual de Montes Claros.

\begin{abstract}
This study sought to evaluate the comprehensive nature of Primary Health Care (PHC), from the standpoint of the users of the Family Health Strategy (FHS) and users of others services of PHC. It involved a cross-sectional, analytical and quantitative study conducted in Montes Claros in the Brazilian state of Minas Gerais. For data collection, the adult version of a validated Primary Care Assessment Tool questionnaire was applied to 373 adult service users, of which 124 (33.6\%) reported attendance by the FHS and 249 (66.4\%) reported attendance by other services. Scores were assigned for the eight dimensions of the instrument, though only three were used for this study, namely first contact, accessibility, and longitudinality. The results showed that in relation to the comprehensive nature of $\mathrm{PHC}$, there was a better evaluation by the population that uses the FHS compared to those who use other services. The first contact, accessibility and longitudinality attributes obtained higher scores in the FHS care provided in comparison with other services, with statistical significance for all attributes. The conclusion drawn is that it is of fundamental importance to assess the attributes of PHC in order to improve the quality of services provided.

Key words Comprehensive care, Primary health care, Evaluation, Users of health services
\end{abstract}

Resumo Este estudo objetivou avaliar o processo de integralidade da Atenção Primária à Saúde (APS), sob o ponto de vista dos usuários da Estratégia Saúde da Família (ESF) e dos demais serviços da APS. Estudo transversal, analítico e quantitativo, realizado em Montes Claros, Minas Gerais, Brasil, que utilizou questionário validado Primary Care Assessment Tool, versão adulto, aplicado a 373 usuários adultos, sendo que 124 (33,6\%) referiram atendimento pela ESF e 249 (66,4\%) referiram atendimento por outros serviços. Atribuíram-se escores para as oito dimensões do instrumento, entretanto, o presente estudo trabalhou com apenas três: primeiro contato; acessibilidade; $e$, longitudinalidade. Em relação à integralidade da APS, houve melhor avaliação pela população que utiliza a ESF, comparando com a de quem utiliza outros serviços. Os atributos primeiro contato, acessibilidade e longitudinalidade obtiveram escores mais elevados na modalidade de ESF em comparação com outros serviços, com significância estatística para todos os domínios. Conclui-se que é de fundamental importância avaliar os atributos da APS, com vistas à melhoria da qualidade dos serviços prestados.

Palavras-chave Integralidade, Atenção Primária à Saúde, Avaliação, Usuários de serviços de saúde 


\section{Introdução}

A inserção de programas inovadores de assistência à saúde no Brasil, durante a década de 1990, elevou a Atenção Primária à Saúde (APS) a uma posição de destaque ${ }^{1}$. A Atenção Primária à Saúde é definida como o primeiro nível de assistência dentro do sistema de saúde, caracterizando-se, principalmente, pela continuidade e integralidade da atenção, além da coordenação da assistência dentro do próprio sistema, da atenção centrada na família, da orientação e participação comunitária e da competência cultural dos profissionais ${ }^{2}$.

O conceito da APS foi sistematizado por Bárbara Starfield ${ }^{2}$ através de atributos denominados essenciais: acesso de primeiro contato, que é a acessibilidade e uso do serviço a cada novo problema ou novo episódio de um problema ${ }^{2}$; a longitudinalidade, que pressupõe a existência de uma fonte regular de atenção e seu uso ao longo do tempo ${ }^{2}$; a integralidade, que implica fazer arranjos para que o paciente receba todos os tipos de serviços de atenção à saúde ${ }^{2}$ e a coordenação, que é a disponibilidade de informações a respeito de problemas $e$ serviços anteriores e o reconhecimento daquela informação na medida em que está relacionada às necessidades para o presente atendimento ${ }^{2}$. Além desses, a autora também define três atributos derivados: a orientação familiar, decorrente da consideração do contexto familiar na atenção integral; a orientação comunitária, que procede do reconhecimento das necessidades sociais; e a competência cultural, que envolve a atenção às necessidades de uma população com características culturais especiais.

A persistência do modelo biomédico e a lacuna que se observa sobre a adesão aos atributos da APS tornam imprescindível a discussão sobre a formação de recursos humanos para o Sistema Único de Saúde (SUS), buscando melhores alternativas para se ter garantias de que as práticas atendam aos desafios necessários para a implementação de uma APS de qualidade².

Estudos apresentam evidências sobre a associação entre o maior grau de orientação à APS e o aumento da efetividade dos sistemas de saúde, satisfação dos usuários, promoção da equidade, integralidade e eficiência ${ }^{3}$. Apesar de a maior parte dos estudos terem sidos realizados em países desenvolvidos, também existem evidências do impacto positivo da APS nos países em desenvolvimento, notadamente nos latino-americanos ${ }^{4,5}$.
A estratégia adotada pelo Ministério da Saúde no Brasil para a expansão da APS e reorientação do SUS é a Estratégia Saúde da Família (ESF), criada em 1994. O financiamento para a expansão da ESF vem crescendo, assim como o número de equipes implantadas ${ }^{6}$. Desse modo, várias iniciativas com foco na avaliação da ESF vêm sendo desenvolvidas no Brasil desde sua criação ${ }^{1,7}$.

Muitas pesquisas ${ }^{8-12}$ mostram o impacto positivo da ESF em relação aos outros serviços da APS sobre o sistema de saúde brasileiro, todavia, os estudos que levam em conta a satisfação do usuário dos serviços da APS (ESF e demais serviços) ainda são escassos e não dispõem de instrumentos de fácil aplicação para uso rotineiro, apesar do incentivo do Ministério da Saúde a pesquisas voltadas para avaliações dos serviços de saúde, objetivando nortear a elaboração e a reorientação de políticas e programas na avaliação da ESF desde a perspectiva dos usuários ${ }^{7}$.

Para a avaliação da APS, a literatura científica dispõe de alguns instrumentos, enfatizando a aferição da presença e extensão dos atributos essenciais e derivados da atenção primária à saú$\mathrm{de}^{13,14}$. Dentre os instrumentos, tem-se o Primary Care Assessment Tool (PCATool), que foi criado nas versões Child Consumer/Client Survey e Adult Consumer/Client Survey, aplicáveis aos usuários para a avaliação da atenção à saúde da criança e do adulto, respectivamente, e Facility/Provider Survey, aplicável aos profissionais de saúde, cujo objetivo é medir a presença e a extensão de cada atributo da $\mathrm{APS}^{2}$. O instrumento já foi validado no Brasil ${ }^{15}$ e produz escores para cada atributo e também os Escores Essencial e Geral, possibilitando mensurar o grau de orientação à APS em diferentes serviços e sistemas de saúde².

A realização de pesquisas que avaliem os serviços da Atenção Primária à Saúde, levando em conta a ótica dos usuários, é essencial e contribui para a institucionalização da avaliação e reorganização dos serviços, além de se constituir em uma ferramenta para a participação popular?

Assim, o objetivo deste estudo foi avaliar o processo de integralidade da Atenção Primária à Saúde em um município ao norte de Minas Gerais, Brasil, a partir de seus atributos, sob o ponto de vista dos usuários da Estratégia Saúde da Família e dos demais serviços da APS. Procede-se, complementarmente, à comparação dos serviços na APS presente no município estudado entre os dois grupos avaliados. 
Trata-se de uma investigação transversal, exploratória, analítica e quantitativa ${ }^{16}$. A pesquisa foi realizada através de um inquérito junto à população atendida pela Estratégia Saúde da Família e demais serviços de APS no município de Montes Claros, ao norte do estado de Minas Gerais, Brasil. Montes Claros é uma cidade polo, com população estimada em 365.000 habitantes. No que tange aos serviços de Atenção Primária à Saúde, à época da coleta de dados (fim de 2012 e início de 2013), o município dispunha de 76 equipes de saúde da família, 10 localizados na zona rural e 66 na urbana; uma unidade de saúde com o Programa de Agentes Comunitários de Saúde (PACS) e 15 Centros de Saúde, sendo estes últimos responsáveis pela assistência à população sem cobertura da Estratégia Saúde da Família (ESF).

A população estudada foi constituída por usuários residentes e cadastrados nas áreas de abrangência das equipes da ESF do município e demais unidades de APS. Foram incluídas apenas as equipes localizadas na zona urbana por limitações logísticas. Os critérios de elegibilidade dos sujeitos para a aplicação do questionário foram: ser usuário há mais de um ano dos serviços de APS (ESF ou outros serviços); ser maior de 18 anos; aceitar participar do estudo. Os usuários foram selecionados através de amostragem aleatória simples. Inicialmente atribuiu-se um número a cada família. Em seguida, procedeu-se ao sorteio, a partir da tabela de números aleatórios e do número de usuários definido pelo cálculo amostral.

O cálculo amostral foi realizado tendo como base uma população finita, com a seguinte fórmula: $n=N \cdot p \cdot q \cdot z^{2} /\left[p \cdot q \cdot z^{2}+(N-1) \cdot e^{2}\right]$, em que "n" é o tamanho da amostra, "N" é o tamanho da população; "p" é igual a 0,5 (proporção populacional de indivíduos que pertence à categoria que estamos interessados em estudar); "q" é igual a 0,5 (proporção populacional de indivíduos que $\mathrm{NÃO}$ pertence à categoria que estamos interessados em estudar $(q=1-p)$; “ $Z$ ” é igual a 1,96 (valor crítico que corresponde ao grau de confiança desejado); "E" é igual a 0,05 (5\%) (margem de erro ou erro máximo de estimativa, que identifica a diferença máxima entre a proporção amostral e a verdadeira proporção populacional [p]).

O cálculo amostral definiu a necessidade de alocação de 373 usuários, considerando-se um erro amostral de 5\% e nível de confiança de 95\%, a partir de uma prevalência do evento estudado de 50\% (utilização do serviço de saúde). Esse va- lor é habitualmente tomado como uma estimativa conservadora, pois aumenta o número da amostra.

O questionário utilizado em sua versão adulto constitui-se em uma adaptação dos instrumentos componentes do Primary Care Assessment Tool, elaborado na Universidade de John Hopkins, aplicado inicialmente no Brasil em $2004^{17}$, e utilizado posteriormente em estudo realizado no Estado do Rio de Janeiro ${ }^{18}$.

Nessa perspectiva, a qualidade da atenção à saúde está sendo entendida, no presente estudo, como a presença e a extensão dos atributos considerados essenciais e derivados da $\mathrm{APS}^{2}$. O PCATool mede a presença e a extensão dos quatro atributos essenciais e dos três derivados da atenção primária e o grau de afiliação do usuário ao serviço de saúde. Esse instrumento, originalmente formado por 77 perguntas (itens) sobre os sete atributos da atenção primária à saúde, possibilita, por meio de respostas tipo Likert, construir escores de Atenção Primária à Saúde com intervalo de 1-4 para cada atributo. O escore final de cada um destes atributos é dado pela média das respostas de seus itens, que também variam de 1-4. Alguns atributos são formados por subdimensões, como acesso (primeiro contato e utilização), integralidade (serviços recebidos e serviços disponíveis) e coordenação (sistema de informação e fluxo de usuários $)^{19}$.

Os dados obtidos a partir dos questionários de usuários foram consolidados com a finalidade de calcular as proporções de respostas positivas (sempre); intermediárias (quase sempre, muitas vezes e algumas vezes, quase nunca) e negativas (nunca), estabelecendo comparações entre as modalidades de organização das ações em saúde: usuários vinculados a uma equipe da ESF e usuários sem vínculo com a ESF (usuários de outros serviços de APS).

Foi calculado o escore geral da APS (valor médio dos atributos essenciais e derivados e o do grau de afiliação). O processamento e a análise dos dados foram realizados através do software SPSS for Windows, versão 18.0. Após a consolidação dos dados relativos a cada atributo, os valores foram transformados em uma escala contínua, variando entre zero e dez, utilizando a fórmula a seguir:

- Escore ajustado $=[($ escore obtido -1$) /(4-$ 1)] x 10 .

- Valores de escores $\geq 6,6$ foram definidos como elevados e equivalentes ao valor três ou mais na escala Likert $^{19}$. Valores $<6,6$ foram considerados baixos. 
Para verificar a significância estatística nas comparações entre proporções, foi realizado o teste de qui-quadrado, com tolerância de erro ao aceitar as diferenças significantes de até $5 \%$. Utilizou-se, ainda, o teste $t$ de student para as médias dos escores dos domínios abordados nos questionários na tabela dos usuários. A descrição dos resultados foi sistematizada, segundo as categorias de análise: primeiro contato, acessibilidade e longitudinalidade.

Os questionários foram aplicados nos domicílios por três enfermeiros previamente treinados. Os usuários foram informados quanto aos objetivos da pesquisa e solicitados a ler e assinar o Termo de Consentimento Livre e Esclarecido. O presente trabalho foi aprovado pelo Comitê de Ética em Pesquisa da Universidade Federal de São Paulo.

\section{Resultados}

Foram entrevistados 373 usuários das unidades de APS incluídas no estudo, sendo que 124 $(33,6 \%)$ se referiram a atendimento pela modalidade de ESF, enquanto 249 (66,4\%) fizeram referência ao uso de outros serviços de APS.

A Tabela 1 traz a proporção de usuários que responderam positivamente (sempre) às perguntas do questionário nas categorias de primeiro contato, acessibilidade e longitudinalidade, conforme o modelo das ações de Atenção Primária à Saúde.

No questionamento acerca do Primeiro Contato, nota-se que os percentuais de respostas positivas são maiores no modelo da ESF em todas as três questões, com significância estatística para todas $(\mathrm{p}=0,005 ; \mathrm{p}=0,001 ; \mathrm{p}=0,014)$ (Tabela 1$)$.

Em relação à Acessibilidade, a maior parte das questões obteve percentuais mais elevados de respostas positivas para os usuários da ESF. As questões que obtiveram percentuais maiores de respostas positivas para os usuários de outros serviços foram as relacionadas a "serviço de saúde ficar aberto no sábado ou no domingo" (ESF $=20,5 \%$; outros serviços $=21,3 \%[\mathrm{p}=0,000])$; "usuário ter que esperar mais de 30 minutos para consulta quando chega no seu serviço de saúde" (ESF $=37,5 \%$; outros serviços $=45,7 \%[\mathrm{p}=0,027]$ ); "usuário ter que esperar por muito tempo, ou falar com muitas pessoas para marcar hora no seu do serviço de saúde" (ESF = 17,4\%; outros serviços $=28,3 \%[\mathrm{p}=0,018])$; "dificuldades em conseguir atendimento médico do serviço de saúde quando pensa que é necessário" (ESF $=11,65 \%$; outros serviços $=25,1 \%[\mathrm{p}=0,000])$; "ter que faltar ao trabalho ou escola para ir ao serviço de saúde" $(E S F=35,5 \%$; outros serviços $=36,8 \%$ [p $=0,339]$ ) (Tabela 1). Essas questões sugerem que o tempo para o acesso a atendimentos nos outros serviços da APS pode ser maior, se comparado ao acesso à ESF, na visão dos usuários.

Quando analisadas as questões sobre a Longitudinalidade (Tabela 1), destaca-se que apenas uma questão recebeu maior percentual de respostas "sempre" para os usuários de outros serviços, que perguntava sobre "mudar do serviço de saúde para outro serviço de saúde se isso fosse muito fácil de fazer" (ESF $=25,6$; Outros serviços $=34,5[\mathrm{p}=0,009])$.

A Tabela 2 apresenta as respostas dos usuários, segundo escores, às questões relacionadas aos atributos primeiro contato, acessibilidade e longitudinalidade.

Com relação ao atributo primeiro contato, observa-se que entre os usuários da ESF há um percentual significativamente maior de avaliações com alto escore $(57,1 \%)$ do que entre os de outros serviços $(45,2 \%)(p=0,037)$. Entretanto, no que diz respeito à acessibilidade, foi observado um percentual de avaliações com alto escore, maior entre usuários de outros serviços $(49,8 \%)$, quando comparado aos usuários da ESF (41,2\%).

Quanto ao atributo longitudinalidade, também observou-se um percentual significativamente maior de avaliações de alto escore entre usuários da $\mathrm{ESF}$ (50,0\%), quando comparado aos de outros serviços de APS $(24,7 \%)(\mathrm{p}<0,001)$.

As médias dos escores dos atributos da APS para a ESF e para os outros tipos de serviço estão dispostas na Tabela 3. A ESF apresenta valores de escores satisfatórios $(\geq 6,6)$ para os atributos de primeiro contato $(7,3)$ e longitudinalidade $(6,9)$. Em contrapartida, os demais serviços de APS apresentam, em todos os atributos avaliados, escores insatisfatórios $(<6,6)$.

\section{Discussão}

Neste estudo, privilegiou-se cada elemento que compõe as dimensões de integralidade analisadas, sendo estas consideradas a partir das respostas a cada uma das questões, e não a partir de um valor sintético de todas de uma mesma dimensão. Isso permitiu visualizar os diferentes aspectos envolvidos no exercício da integralidade e a percepção do usuário dos serviços da APS. Outros estudos ${ }^{17,20}$ realizados em São Paulo, Brasil, que também fizeram uso do PCATool, utilizaram 
Tabela 1. Número e Proporção (\%) de usuários que responderam positivamente (sempre) às perguntas do questionário nas categorias de primeiro contato, acessibilidade e longitudinalidade, conforme o modelo das ações de APS, Montes Claros, 2012.

\begin{tabular}{|c|c|c|c|c|c|}
\hline \multirow[t]{2}{*}{ Dimensão APS } & \multicolumn{2}{|c|}{ ESF } & \multicolumn{2}{|c|}{$\begin{array}{c}\text { Outros } \\
\text { serviços }\end{array}$} & \multirow[b]{2}{*}{$\mathbf{p}$} \\
\hline & $\mathbf{n}$ & $\%$ & $\mathbf{n}$ & $\%$ & \\
\hline \multicolumn{6}{|l|}{ Primeiro Contato } \\
\hline $\begin{array}{l}\text { Quando você necessita de uma consulta de revisão (consulta de rotina, } \\
\text { check-up), você vai ao seu "nome do serviço de saúde/ ou nome do } \\
\text { médico/enfermeiro" antes de ir a outro serviço de saúde? }\end{array}$ & 51 & 41,8 & 61 & 25,0 & 0,005 \\
\hline $\begin{array}{l}\text { Quando você tem um problema de saúde, você vai ao seu "nome do } \\
\text { serviço de saúde/ ou nome do médico/enfermeiro" antes de ir a outro } \\
\text { serviço de saúde? }\end{array}$ & 51 & 42,1 & 52 & 21,3 & 0,001 \\
\hline $\begin{array}{l}\text { Quando você tem que consultar um especialista, o seu "nome do } \\
\text { serviço de saúde/ ou nome do médico/enfermeiro" tem que encaminhar } \\
\text { você obrigatoriamente? }\end{array}$ & 33 & 21,5 & 40 & 16,5 & 0,014 \\
\hline \multicolumn{6}{|l|}{ Acessibilidade } \\
\hline $\begin{array}{l}\text { O "nome do serviço de saúde/ ou nome do médico/enfermeiro" fica } \\
\text { aberto no sábado ou no domingo? }\end{array}$ & 25 & 20,5 & 52 & 21,3 & 0,000 \\
\hline $\begin{array}{l}\text { O "nome do serviço de saúde/ ou nome do médico/enfermeiro" fica } \\
\text { aberto pelo menos algumas noites de dias úteis até às } 20 \text { horas? }\end{array}$ & 32 & 26,4 & 48 & 19,7 & 0,000 \\
\hline $\begin{array}{l}\text { Quando o seu "nome do serviço de saúde/ ou nome do médico/ } \\
\text { enfermeiro" está aberto e você adoece alguém de lá atende você no } \\
\text { mesmo dia? }\end{array}$ & 47 & 38,8 & 53 & 21,8 & 0,000 \\
\hline $\begin{array}{l}\text { Quando o seu "nome do serviço de saúde/ ou nome do médico/ } \\
\text { enfermeiro" está aberto, você consegue aconselhamento rápido pelo } \\
\text { telefone se precisar? }\end{array}$ & 28 & 23,1 & 28 & 11,5 & 0,064 \\
\hline $\begin{array}{l}\text { Quando o seu "nome do serviço de saúde/ ou nome do médico/ } \\
\text { enfermeiro" está aberto, existe um número de telefone para o qual você } \\
\text { possa ligar quando fica doente? }\end{array}$ & 39 & 32,3 & 50 & 20,5 & 0,013 \\
\hline $\begin{array}{l}\text { Quando o seu "nome do serviço de saúde/ ou nome do médico/ } \\
\text { enfermeiro" está fechado no sábado e no domingo e você fica doente, } \\
\text { alguém deste serviço atende você no mesmo dia? }\end{array}$ & 27 & 22,3 & 29 & 11,8 & 0,000 \\
\hline $\begin{array}{l}\text { Quando o seu "nome do serviço de saúde/ ou nome do médico/ } \\
\text { enfermeiro" está fechado e você fica doente durante a noite, alguém } \\
\text { deste serviço atende você naquela noite? }\end{array}$ & 31 & 25,8 & 24 & 9,8 & 0,000 \\
\hline $\begin{array}{l}\text { É fácil marcar hora para uma consulta de revisão (consulta de rotina, } \\
\text { "check-up") neste "nome do serviço de saúde/ ou nome do médico/ } \\
\text { enfermeiro"? }\end{array}$ & 41 & 33,9 & 30 & 12,3 & 0,014 \\
\hline $\begin{array}{l}\text { Quando você chega no seu "nome do serviço de saúde/ ou nome } \\
\text { do médico/enfermeiro", você tem que esperar mais de } 30 \text { minutos } \\
\text { para consultar com o médico ou enfermeiro (sem contar triagem ou } \\
\text { acolhimento? }\end{array}$ & 45 & 37,5 & 111 & 45,7 & 0,027 \\
\hline $\begin{array}{l}\text { Você tem que esperar por muito tempo, ou falar com muitas pessoas } \\
\text { para marcar hora no seu "nome do serviço de saúde/ ou nome do } \\
\text { médico/enfermeiro"? }\end{array}$ & 21 & 17,4 & 69 & 28,3 & 0,018 \\
\hline $\begin{array}{l}\text { É difícil para você conseguir atendimento médico do "nome do serviço } \\
\text { de saúde/ ou nome do médico/enfermeiro" quando pensa que é } \\
\text { necessário? }\end{array}$ & 14 & 11,6 & 61 & 25,1 & 0,000 \\
\hline $\begin{array}{l}\text { Quando você tem que ir ao "nome do serviço de saúde/ ou nome do } \\
\text { médico/enfermeiro", você tem que faltar ao trabalho ou à escola para ir } \\
\text { ao serviço de saúde? }\end{array}$ & 43 & 35,5 & 89 & 36,8 & 0,339 \\
\hline
\end{tabular}

estratégia diferente, chegando a um escore sintético de cada dimensão analisada.
De modo geral, a avaliação da integralidade pelos usuários da ESF se mostrou favorável nas 
Tabela 1. continuação

\begin{tabular}{|c|c|c|c|c|c|}
\hline \multirow[t]{2}{*}{ Dimensão APS } & \multicolumn{2}{|c|}{ ESF } & \multicolumn{2}{|c|}{$\begin{array}{c}\text { Outros } \\
\text { serviços }\end{array}$} & \multirow[b]{2}{*}{$\mathbf{p}$} \\
\hline & $\mathbf{n}$ & $\%$ & n & $\%$ & \\
\hline \multicolumn{6}{|l|}{ Longitudinalidade } \\
\hline $\begin{array}{l}\text { Quando você vai ao seu "nome do serviço de saúde/ ou nome do } \\
\text { médico/enfermeiro", é o mesmo médico ou enfermeiro que atende você } \\
\text { todas as vezes? }\end{array}$ & 10 & 8,1 & 8 & 3,3 & 0,070 \\
\hline $\begin{array}{l}\text { Você acha que o seu "médico/enfermeiro" entende o que você diz ou } \\
\text { pergunta? }\end{array}$ & 18 & 14,6 & 14 & 5,7 & 0,012 \\
\hline $\begin{array}{l}\text { O seu "médico/enfermeiro" responde suas perguntas de maneira que } \\
\text { você entenda? }\end{array}$ & 20 & 16,4 & 23 & 9,3 & 0,096 \\
\hline $\begin{array}{l}\text { Se você tiver alguma pergunta, pode telefonar e falar com o médico ou } \\
\text { enfermeiro que melhor conhece você? }\end{array}$ & 12 & 9,8 & 9 & 3,7 & 0,072 \\
\hline $\begin{array}{l}\text { O seu "médico/enfermeiro" lhe dá tempo suficiente para falar sobre suas } \\
\text { preocupações ou problemas? }\end{array}$ & 51 & 43,2 & 41 & 17,4 & 0,000 \\
\hline $\begin{array}{l}\text { Você se sente à vontade contando suas preocupações ao seu "médico/ } \\
\text { enfermeiro"? }\end{array}$ & 52 & 44,1 & 41 & 17,4 & 0,000 \\
\hline $\begin{array}{l}\text { O seu "médico/enfermeiro" conhece mais você como pessoa do que } \\
\text { somente como alguém com um problema de saúde? }\end{array}$ & 34 & 28,8 & 28 & 11,8 & 0,001 \\
\hline O seu "médico/enfermeiro" sabe quem mora com você? & 37 & 31,1 & 27 & 11,4 & 0,000 \\
\hline $\begin{array}{l}\text { O seu "médico/enfermeiro" sabe quais problemas são mais importantes } \\
\text { para você?? }\end{array}$ & 22 & 18,6 & 17 & 7,2 & 0,002 \\
\hline $\begin{array}{l}\text { O seu "médico/enfermeiro" conhece sua história clínica (médica) } \\
\text { completa? }\end{array}$ & 35 & 29,4 & 21 & 8,9 & 0,001 \\
\hline O seu "médico/enfermeiro" sabe a respeito do seu trabalho ou emprego? & 35 & 29,9 & 26 & 11,1 & 0,011 \\
\hline $\begin{array}{l}\text { O seu "médico/enfermeiro" saberia de alguma forma se você tivesse } \\
\text { problemas em obter ou pagar por medicamentos que você precisa? }\end{array}$ & 17 & 14,5 & 25 & 10,6 & 0,004 \\
\hline $\begin{array}{l}\text { O seu "médico/enfermeiro" sabe a respeito de todos os medicamentos } \\
\text { que você está tomando? }\end{array}$ & 33 & 28,2 & 31 & 13,2 & 0,000 \\
\hline $\begin{array}{l}\text { Você mudaria do "nome do serviço de saúde/ ou nome médico/ } \\
\text { enfermeiro" para outro serviço de saúde se isto fosse muito fácil de } \\
\text { fazer? }\end{array}$ & 30 & 25,6 & 82 & 34,5 & 0,009 \\
\hline
\end{tabular}

*Teste do qui-quadrado para proporções. Proporção dos usuários que afirmaram COM CERTEZA SIM na utilização dos referidos serviços (n e \%).

Tabela 2. Número e Proporção (\%) de usuários que responderam, segundo escores, às perguntas do questionário nas categorias de primeiro contato, acessibilidade e longitudinalidade, conforme o modelo das ações de APS, Montes Claros, 2012.

\begin{tabular}{|c|c|c|c|c|c|c|c|c|c|}
\hline \multirow{3}{*}{ Atributo da APS } & \multicolumn{4}{|c|}{ ESF } & \multicolumn{4}{|c|}{ Outros serviços } & \multirow{3}{*}{$\mathbf{p}$} \\
\hline & \multicolumn{2}{|c|}{$\begin{array}{c}\text { Baixo escore } \\
(<6,6)\end{array}$} & \multicolumn{2}{|c|}{$\begin{array}{l}\text { Alto escore } \\
(\geq 6,6)\end{array}$} & \multicolumn{2}{|c|}{$\begin{array}{c}\text { Baixo escore } \\
(<6,6)\end{array}$} & \multicolumn{2}{|c|}{$\begin{array}{l}\text { Alto escore } \\
\quad(\geq 6,6)\end{array}$} & \\
\hline & $\mathrm{n}$ & $\%$ & $\mathbf{n}$ & $\%$ & $\mathrm{n}$ & $\%$ & $\mathbf{n}$ & $\%$ & \\
\hline Primeiro Contato & 36 & 42,9 & 48 & 57,1 & 142 & 54,8 & 117 & 45,2 & 0,037 \\
\hline Acessibilidade & 50 & 58,8 & 35 & 41,2 & 131 & 50,2 & 130 & 49,8 & 0,104 \\
\hline Longitudinalidade & 41 & 50,0 & 41 & 50,0 & 189 & 75,3 & 62 & 24,7 & $<0,001$ \\
\hline
\end{tabular}

Teste qui-quadrado de Pearson.

questões que compõem o primeiro contato, a longitudinalidade e a acessibilidade, pois, mesmo nos questionamentos que obtiveram mais respostas positivas nas questões da acessibilidade para os usuários de outro serviço, sugeriu-se que o tempo para o acesso aos outros serviços da APS 
Tabela 3. Média de escores quanto às categorias de primeiro contato, acessibilidade e longitudinalidade, Montes Claros, 2012.

\begin{tabular}{lccccccr}
\hline \multirow{2}{*}{ Atributo da APS } & \multicolumn{2}{c}{ ESF } & & \multicolumn{2}{c}{ Outros serviços } & & p \\
\cline { 2 - 3 } & Média & DP & & Média & DP & & 0,001 \\
\hline Primeiro Contato & 7,3 & 2,3 & & 5,9 & 2,5 & $<0,001$ \\
Acessibilidade & 5,4 & 2,1 & & 4,3 & 1,9 & $<0,001$ \\
Longitudinalidade & 6,9 & 1,9 & & 4,7 & 2,1 & \\
\hline
\end{tabular}

Teste t de student.

é maior do que o tempo na ESF. Corroborando, estudo realizado em São Paulo que encontrou o mesmo achado para essas dimensões ${ }^{21}$.

Ao analisar-se a acessibilidade, os resultados apontaram para as dificuldades de rápido acesso a outros serviços da APS, fato que pode impactar negativamente na avaliação do usuário sobre o serviço, em especial no que diz respeito à resolução de queixas agudas ou subagudas ${ }^{21}$.

Quanto aos escores calculados a partir da avaliação, notou-se que a ESF apresentou escores maiores em "primeiro contato", "acessibilidade" e "longitudinalidade" do que aqueles atribuídos às demais unidades de APS, assim como em outros estudos $^{17,19,22}$.

No que diz respeito ao acesso aos serviços de saúde, a APS, como serviço de primeiro contato, articulado a outros níveis, pressupõe a constituição de uma rede integrada de serviços de saúde, funcionando como porta de entrada no sistema para o atendimento, ao longo do tempo, às necessidades e problemas de saúde das pessoas (não relacionados apenas à enfermidade), coordenando ou integrando os outros tipos de atenção, fornecidos em algum ponto do sistema de saúde. A garantia de atenção ao primeiro contato está relacionada ao uso da APS como a porta de entrada para as questões de saúde e à sua capacidade para lidar com problemas amplos, heterogêneos e fortemente influenciados pelo contexto social ${ }^{2}$.

Nesse ínterim, a dimensão do acesso traduz principalmente a maior ou menor facilidade da população para obter uma consulta. Ainda que não devamos reduzir a atenção primária à prestação de consultas médicas, ela não pode abdicar da atenção clínica prestada pelos profissionais de saúde ${ }^{22}$.

No presente estudo foi registrado um menor escore no que tange à acessibilidade (média de escore $=5,4$ ), fato que constitui um enorme desafio na busca da integralidade da atenção à saúde $^{17,20,23}$. Outros autores ${ }^{24}$ apontam em seus estudos que unidades de ESF, muitas vezes, apre- sentam desempenho semelhante ao modelo tradicional no que se refere à acessibilidade e porta de entrada, revelando diversidade na operacionalização do referido programa.

Estudos realizados no município de São Pau$1 \mathrm{o}^{20} \mathrm{e}$ em outros acima de 100 mil habitantes no Estado de São Paulo ${ }^{17}$ também encontraram escores intermediários na avaliação da acessibilidade pelos usuários.

Nesse contexto, a oferta de serviços e sua distribuição geográfica, a disponibilidade e a qualidade dos recursos humanos e tecnológicos, os mecanismos de financiamento, o modelo assistencial e a informação acerca do sistema são características da oferta que afetam o acesso ${ }^{17}$. A dimensão do acesso diz respeito principalmente à maior ou menor facilidade da população para obter uma consulta ${ }^{22}$.

Em relação ao atributo longitudinalidade, em que é avaliada, acima de tudo, a relação entre usuário e profissional, no presente estudo houve uma boa avaliação (alto escore) por parte dos usuários da ESF, sendo que o mesmo não é observado entre os usuários dos demais serviços de APS. É reconhecido na literatura que a produção da saúde demanda o estabelecimento de relações de intersubjetividade entre profissionais e usuários, abrindo-se a possibilidade de aproximação ao mundo da vida dos pacientes, portanto ao seu modo de adoecer e morrer ${ }^{22}$. Vale salientar, ainda, que a presença adequada da longitudinalidade é um fator essencial para o sistema de saúde, pois esse atributo tende a produzir diagnósticos $\mathrm{e}$ tratamentos mais precisos, além da redução dos encaminhamentos desnecessários para especialistas e para a realização de procedimentos mais complexos ${ }^{25}$.

Contudo, a melhoria dos serviços prestados nesse nível de atenção ainda é um desafio, apesar do avanço do Sistema Único de Saúde no processo de reorganização da atenção básica, inclusive com a ampliação da cobertura e implementação de novos modelos assistenciais. Nesse aspecto, 
posto que estudos relacionam a longitudinalidade/vínculo longitudinal com resultados positivos, o reconhecimento desse atributo como característica central da APS em nosso país é oportuno e deve ser almejado e avaliado ${ }^{26,27}$.

Ademais, algumas limitações podem ser reconhecidas neste estudo. O instrumento PCATool, inclusive, apresenta limitações. A primeira é considerar, para o cálculo dos Escores Essencial e Geral, que os atributos tenham os mesmos "pesos" na medida da orientação à APS. A segunda consiste no fato de o estudo ser conduzido em um único município. Por último, a APS foi avaliada sob a ótica dos usuários, e alguns autores ${ }^{28}$ salientam que há certa "naturalização" para a baixa qualidade na visão dos usuários, os quais terminam por reconhecer o serviço recebido como um favor ou doação e não como um direito, o que representa uma das diferentes interpretações para o gratuito. Além disso, esta é uma pesquisa avaliativa, transversal, sujeita às limitações próprias deste tipo de delineamento.

Os resultados apresentados neste estudo trazem importantes implicações para os serviços avaliados e também para as políticas públicas de atenção à saúde na microrregião. Porém, alguns atributos, como acessibilidade, na experiência dos usuários, precisam ser melhorados. Além disso, é registrada uma significativa diferença na avaliação feita pelos usuários da ESF, quando comparada aos dos demais serviços de APS.
Ademais, a avaliação da satisfação dos usuários dos serviços de saúde, em todos os âmbitos da atenção, constitui uma ferramenta importante que subsidia o processo de decisão compartilhada, de forma a se repensar as práticas profissionais, reorganizar o processo de trabalho desenvolvido, realocar recursos, readequar ações e redefinir objetivos que estejam coerentes com o projeto de saúde estabelecido.

Nesse sentido, os processos de avaliação da satisfação dos usuários nos serviços públicos de saúde ajudam na construção de uma nova perspectiva do cuidado e, além de monitorar as atividades desses serviços, podem fortalecer o controle social e a participação/inclusão dos usuários nos processos de planejamento. Os Processos de avaliação podem, então, contribuir para a construção de alternativas conjuntas - entre serviços de saúde e usuários - para intervenções mais adequadas que possam solucionar problemas presentes no cotidiano dos serviços, propiciando avanços no âmbito da produção de cuidados e gestão dos serviços de saúde.

Por conseguinte, o presente trabalho foi capaz de demonstrar a importância de se avaliar os atributos da APS nas distintas modalidades de cuidado sob a perspectiva do usuário, principalmente pela aplicabilidade prática do instrumento utilizado na condução do estudo, representando importante ferramenta para a melhoria da qualidade dos serviços de saúde.

\section{Colaboradores}

CSO Silva trabalhou na concepção, coleta, análise e interpretação dos dados, redação do artigo; ADG Fonseca e LP Souza e Souza trabalharam na coleta, análise e interpretação dos dados, e redação do artigo. LG Siqueira, AGS Belasco trabalharam na análise e interpretação dos dados, e redação do artigo. DA Barbosa trabalhou na análise e interpretação dos dados, redação do artigo e a sua revisão crítica. 


\section{Referências}

1. Conill EM. Ensaio histórico-conceitual sobre a Atenção Primária à Saúde: desafios para a organização de serviços básicos e da Estratégia Saúde da Família em centros urbanos no Brasil. Cad Saude Publica 2008, 24(Supl. 1):7-27.

2. Starfield B, organizador. Atenção primária: equilíbrio entre necessidades de saúde, serviços e tecnologia. Brasília: Organização das Nações Unidas para a Educação, a Ciência e a Cultura, Ministério da Saúde; 2002.

3. Organización Panamericana de la Salud, Organización Mundial de la Salud. La renovación de la atención primaria de salud en las Américas. Washington: Organización Panamericana de la Salud, Organización Mundial de la Salud; 2007.

4. Starfield B, Shi L, Macinko J. Contribution of primary care to health systems and health. Milbank Q 2005; 83(3):457-502.

5. Macinko J, Starfield B, Erinosho T. The impact of primary healthcare on population health in low and middle income countries. J Ambul Care Manage 2009; 32(2):150-171.

6. Moore D, Castillo E, Richardson C, Reid R. Determinants of health status and the influence of primary health care services in Latin America, 1990-98. Int J Health Plann Manage 2003; 18(4):279-292.

7. Felisberto E. Da teoria à formulação de uma Política Nacional de Avaliação em Saúde: reabrindo o debate. Cien Saude Colet 2006; 11(3):553-563.

8. Macinko J, Guanais F, Souza M. Evaluation of the impact of Family Health Program on infant mortality in Brazil, 1990-2002. J Epidemiol Community Health 2006; 60(1):13-19.

9. Aquino R, Oliveira N, Barreto M. Impact of the family health program on infant mortality in Brazilian municipalities. Am J Public Health 2008; 99(1):87-93.

10. Facchini L, Piccini R, Tomasi E, Silveira E, Siqueira F, Rodrigues M. Desempenho do PSF no Sul e no Nordeste do Brasil: avaliação institucional e epidemiológica da Atenção Básica à Saúde. Cien Saude Colet 2006; 11(3):669-681.

11. Roncalli A, Lima K. Impacto do PSF sobre indicadores de saúde da criança em municípios de grande porte do Nordeste do Brasil. Cien Saude Colet 2006; 11(3):713724.

12. Rocha PM, Uchoa AC, Rocha NSPD, Souza ECF, Rocha ML, Pinheiro TXA. Avaliação do Programa Saúde da Família em municípios do Nordeste brasileiro: velhos e novos desafios. Cad Saude Publica 2008; 24(Supl. 1):S69-78

13. Shi L, Starfield B, Jiahong X. Validating the adult primary care assessment Tool. J Fam Pract 2001; 50(2): 161-175.

14. Cassady CE, Starfield B, Hurtado MP, Berk RA, Nanda JP, Friedenberg LA. Clinical care for children: measuring consumer experiences with primary care. Pediatrics 2000; 105(3):998-1003.

15. Harzheim E, Starfield B, Rajmil L, Álvarez-Dardet C, Stein AT. Consistência interna e confiabilidade da versão em português do Instrumento de Avaliação da Atenção Primária (PCATool-Brasil) para serviços de saúde infantil. Cad Saude Publica 2006; 22(8):16491659.

16. Pereira MG. Epidemiologia. Teoria e Prática. $4^{a}$ edição. Rio de Janeiro: Guanabara-Koong; 2000.
17. Ibañez N, Rocha JSY, Castro PC, Ribeiro MCSA, Forster AC, Novaes MHD, Viana ALD. Avaliação do desempenho da atenção básica no Estado de São Paulo. Cien Saude Colet 2006; 11(3):683-703.

18. Almeida CM. Validação de uma metodologia de avaliação rápida das características organizacionais e do desempenho de serviços de atenção básica do SUS em nível local [relatório final]. Rio de Janeiro: Fiocruz; 2004.

19. Figueiredo AM, Kuchenbecker RS, Harzheim E, Vigo A, Hauser L, Chomatas ERV. Análise de concordância entre instrumentos de avaliação da Atenção Primária à Saúde na cidade de Curitiba, Paraná, em 2008. Epidemiol Serv Saúde 2013; 22(1):41-48.

20. Elias PE, Ferreira CW, Alves MCG, Cohn A, Kishima V, Escrivão Junior A, Gomes A, Bousquat A. Atenção básica em saúde: comparação entre PSF e UBS por estrato de exclusão social no município de São Paulo. Cien Saude Colet 2006; 11(3):633-641.

21. Sala A, Luppi CG, Simões O, Marsiglia RG. Integralidade e Atenção Primária à Saúde: avaliação na perspectiva dos usuários de unidades de saúde do município de São Paulo. Saude soc 2011; 20(4):948-960.

22. Van Stralen CJ, Belisário SA, Van Stralen TBS, Lima AMD, Massote AW, Oliveira CL. Percepção dos usuários e profissionais de saúde sobre atenção básica: comparação entre unidades com e sem saúde da família na Região Centro-Oeste do Brasil. Cad Saude Publica 2008; 24(Supl. 1):S148-S158.

23. Samico I, Hartz MZA, Felisberto E, Carvalho EF. Atencão à saúde da criança: uma análise do grau de implantação e da satisfação de profissionais e usuários em dois municípios do estado de Pernambuco. Rev Bras Saude Mater Infant 2005; 5(2):229-240.

24. Cordeiro H, Pereira T, Costa H, Pereira ATS. Acreditação como avaliação nos serviços de atenção primária em saúde. In: Hartz Z, Felisberto E, Vieira-da-Silva LM, organizadores. Meta-avaliação da atenção básica da saúde; teoria e prática. Rio de Janeiro: Editora Fiocruz; 2008. p. 153-166.

25. Gérvas J, Fernández MP. El fundamento científico de la función de filtro del médico general. Rev Bras Epidemiol 2005; 8(2):205-218.

26. Cunha EM, Giovanella L. Longitudinalidade/continuidade do cuidado: identificando dimensões e variáveis para a avaliação da Atenção Primária no contexto do sistema público de saúde brasileiro. Cien Saude Colet 2011; 16(Supl. 1):1029-1042.

27. Castro RCL, Knauth DR, Harzheim E, Hauser L, Duncan BBW. Avaliação da qualidade da atenção primária pelos profissionais de saúde: comparação entre diferentes tipos de serviços. Cad Saude Publica 2012; 28(9):1772-1784.

28. Costa GD, Cotta RMM, Franceschini SCC, Batista RS, Gomes AP, Martins PC, Ferreira MLSM. Avaliação em saúde: reflexões inscritas no paradigma sanitário contemporâneo. Physis 2008; 18(4):705-726.

Artigo apresentado em 13/08/2013

Aprovado em 04/01/2014

Versão final apresentada em 06/01/2014 